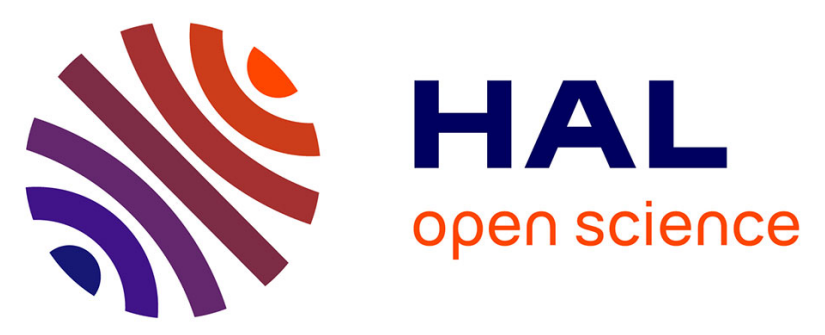

\title{
TDDFT - Generalized kick perturbations and monitoring observables for calculation of excitation energies
}

Robert Hammerling

\section{- To cite this version:}

Robert Hammerling. TDDFT - Generalized kick perturbations and monitoring observables for calculation of excitation energies. Philosophical Magazine, 2008, 88 (18-20), pp.2817-2827. 10.1080/14786430802263137 . hal-00513922

\section{HAL Id: hal-00513922 \\ https://hal.science/hal-00513922}

Submitted on 1 Sep 2010

HAL is a multi-disciplinary open access archive for the deposit and dissemination of scientific research documents, whether they are published or not. The documents may come from teaching and research institutions in France or abroad, or from public or private research centers.
L'archive ouverte pluridisciplinaire HAL, est destinée au dépôt et à la diffusion de documents scientifiques de niveau recherche, publiés ou non, émanant des établissements d'enseignement et de recherche français ou étrangers, des laboratoires publics ou privés. 


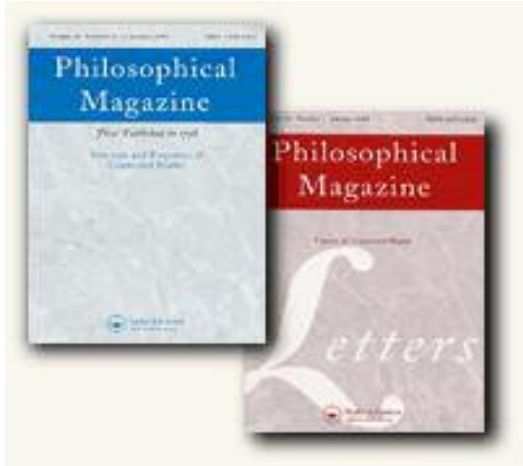

TDDFT - Generalized kick perturbations and monitoring observables for calculation of excitation energies

\begin{tabular}{|r|l|}
\hline Journal: & Philosophical Magazine \& Philosophical Magazine Letters \\
\hline Manuscript ID: & TPHM-08-May-0173.R1 \\
\hline Journal Selection: & Philosophical Magazine \\
\hline $\begin{array}{r}\text { Date Submitted by the } \\
\text { Author: }\end{array}$ & 05-Jun-2008 \\
\hline Komplete List of Authors: & Hammerling, Robert; CMS, TU Wien, E134 \\
\hline Keywords: & density-functional theory, electron energy states, electronic spectra \\
\hline \multicolumn{2}{|c|}{} \\
\hline $\begin{array}{l}\text { Note: The following files were submitted by the author for peer review, but cannot be converted } \\
\text { to PDF. You must view these files (e.g. movies) online. }\end{array}$ & tddft, excitation energies \\
\hline hammerling_ICCMS.tex \\
\hline
\end{tabular}

\section{ScholarONE" \\ Manuscript Central}


Philosophical Magazine

Vol. 00, No. 00, 21 December 2008, 1-11

\title{
RESEARCH ARTICLE
}

\section{TDDFT - Generalized kick perturbations and monitoring observables for calculation of excitation energies}

\author{
Robert Hammerling \\ Email: rh@cms.tuwien.ac.at \\ Center for Computational Materials Science, Vienna University of Technology, \\ Getreidemarkt 9/134, A-1060 Vienna, Austria \\ Wolfgang Pauli Institute, Nordbergstr. 15, A-1090 Wien, Austria
}

( $v 4.1$ released May 2008)

\begin{abstract}
This paper introduces a generalized way to calculate excitation energies of many-body Hamilton operators by propagating kicked orbitals within time dependent density functional theory (TDDFT). We use generalized $\delta(t)$ perturbations and monitor certain observables of the reduced one-body density to calculate the excitation energies of a static many particle problem. This generalizes the usual procedure of a linear momentum kick and monitoring the induced t-dependent dipole moment or higher moments. In particular we propose to monitor the time dependent local charges (e.g. Voronoi,...) in a molecule. As simple application the $S=0$ singlet excitation spectrum of Helium is calculated with this formalism included in a standard TDDFT-software package for atoms, QPROP [1].
\end{abstract}

Keywords: time dependent density functional theory; excitation spectrum; kick perturbation; time dependent local charges

\section{Introduction}

If one is interested in excited state properties of static many-body systems, time dependent density functional theory (TDDFT) [2] can be a useful tool [3]. In particular, in order to calculate approximations to the true excitation energies of the system, TDDFT provides a way to go beyond static Kohn-Sham (KS) eigenvalue differences. In this paper a theory of generalized $\delta(t)$ perturbations, in the following called kick perturbations, and only implicitly t-dependent propagation afterwards is presented. In order to find the values of excited state properties appropriate observables are monitored. Certain new classes of useful observables are presented in this paper. In particular characteristic functions of certain regions, e.g. atomic Voronoi cells, leading to the concept of time dependent local charges are found to be useful. As a simple application of this rather general concept we demonstrate its usefulness on atoms, in particular the $S=0$ singlet excitation spectrum of Helium. We find an improvement for certain excitations compared to the standard TDDFT method, which employs a linear momentum kick and multipole moment monitoring. 


\section{Statement of the many body problem}

Take a $N$ electron system with the time independent many-body Hamilton operator (e.g. Coulombic molecule) defined in the many-body Hilbert space

$$
\begin{aligned}
& \mathcal{H}^{(N)} \cong L^{2}\left(\mathbb{R}^{3 N}\right) \\
& \widehat{H}^{(N)}=\widehat{H}=\widehat{T}+\widehat{V}+\widehat{W},
\end{aligned}
$$

as a self adjoint realization of a linear operator in the Hilbert space $\mathcal{H}^{(N)}$. The operator $\widehat{T}$ is the kinetic energy, $\widehat{V}$ is the external potential and $\widehat{W}$ the two-body interactions. We are interested in solutions of the many-body spectral problem

$$
\widehat{H} \Phi=E \Phi
$$

In the following for ease of notation we pretend that the operator $\widehat{H}$ only has a pure point spectrum of eigenfunctions, but the essence stays the same in the case of non-empty continuous spectrum.

The ground state of the static problem is denoted by

$$
\Phi_{0} \text { with energy } E_{0}
$$

Out of $\Phi_{0}\left(\mathbf{x}_{1}, \ldots, \mathbf{x}_{N}\right)$ we can calculate the many particle density (matrix) and the contracted reduced densities, especially the reduced one particle density $\rho(\mathbf{x})$

$$
\begin{aligned}
\rho^{(N)}\left(\mathbf{x}_{1}, \ldots, \mathbf{x}_{N}\right) & =\left|\Phi_{0}\left(\mathbf{x}_{1}, \ldots, \mathbf{x}_{N}\right)\right|^{2} \\
\gamma^{(N)}\left(\mathbf{x}_{1}, \ldots, \mathbf{x}_{N} ; \mathbf{x}_{1}^{\prime}, \ldots, \mathbf{x}_{N}^{\prime}\right) & =\Phi_{0}\left(\mathbf{x}_{1}, \ldots, \mathbf{x}_{N}\right) \Phi_{0}^{*}\left(\mathbf{x}_{1}^{\prime}, \ldots, \mathbf{x}_{N}^{\prime}\right) \\
\rho(\mathbf{x}) & =N \int d^{N-1} \mathbf{x} \rho^{(N)}\left(\mathbf{x}, \mathbf{x}_{2}, \ldots, \mathbf{x}_{N}\right) \\
\gamma\left(\mathbf{x}, \mathbf{x}^{\prime}\right) & =N \int d^{N-1} \mathbf{x} \gamma^{(N)}\left(\mathbf{x}, \mathbf{x}_{2}, \ldots, \mathbf{x}_{N} ; \mathbf{x}^{\prime}, \mathbf{x}_{2}, \ldots, \mathbf{x}_{N}\right)
\end{aligned}
$$

We denote the excited states by

$$
\Phi_{j} \quad \text { with energy } E_{j}
$$

with some appropriate multiindex $j$. The true excitation energies of the interacting many particle system are denoted by

$$
E_{j, k}=E_{j}-E_{k}=E_{j, 0}-E_{k, 0}
$$

Further the time dependent many particle linear Schrödinger equation initial value problem (IVP) determines the time dependence

$$
\left\{\begin{array}{c}
i \frac{d}{d t} \Psi(t)=\widehat{H}(t) \Psi(t) \\
\Psi\left(t_{0}\right)=\Psi_{0}
\end{array}\right.
$$

Because of the well-known practical inability to solve many body problems directly one uses effective one-body theories with DFT as one of the most prominent representatives. 


\section{The dynamics of the Kohn-Sham system}

Standard static DFT uses an auxiliary non-interacting fermionic $N$ particle system with Hamiltonian

$$
\widehat{H}_{K S}=\widehat{T}+\widehat{V}_{K S}=\sum_{n=1}^{N} \widehat{h}_{K S}
$$

which leads to an effective one particle, but nonlinear spectral problem

$$
\left\{\begin{array}{c}
\widehat{h}_{K S}[\rho] \varphi_{n}=\varepsilon_{n} \varphi_{n} \quad n=1, \ldots, N \\
\rho_{K S}(\mathbf{x})=\sum_{n=1}^{N}\left|\varphi_{n}(\mathbf{x})\right|^{2} \\
v_{K S}[\rho](\mathbf{x})=v_{0}(\mathbf{x})+v_{H}[\rho](\mathbf{x})+v_{X C}[\rho](\mathbf{x})
\end{array}\right.
$$

We recall that static DFT with an exact xc-functional gives the ground state energy $E_{0}$, with an approximate xc-functional one obtains only an approximation to $E_{0}^{(K S)}$. While the density of the interacting and the non-interacting exact KS system is the same, $\rho_{K S}(\mathbf{x})=\rho(\mathbf{x})$, other quantities are in general different.

We assume non-degeneracy of the ground state so that the KS system is the one with the $N$ lowest KS orbitals occupied. Excitations of the noninteracting KS system are described by unoccupied states of the (already determined, frozen) KS Hamiltonian:

$$
\widehat{h}_{K S}[\rho] \varphi_{j}=\varepsilon_{j} \varphi_{j} \quad(j \in\{N+1, \ldots\})
$$

Unoccupied orbitals are calculated but do not enter the KS Hamiltonian $\widehat{h}_{K S}[\rho]$ which is determined by the occupied orbitals only. The excitation energies of the interacting many body system are in general different from the energy differences between unoccupied and occupied KS orbitals. These so called KS single excitation energies of the static KS system are given by

$$
\Delta_{j, j^{\prime}}=\varepsilon_{j}-\varepsilon_{j^{\prime}} \text { with } j>N, j^{\prime} \leq N
$$

and are only zero order approximations [4] of the true excitation energies $E_{j, 0}$. The question if, why and for which systems KS excitation energies are already good approximations has been considered in Ref. [5].

\subsection{Excitation energies with TDDFT}

It is well established that one can use TDDFT to calculate (approximations to) true excitation energies [6].

Within TDDFT there are on the one side a few related methods summarized here by "linear response-TDDFT" and on the other side explicit time propagation methods. These linear response-TDDFT methods start with both the occupied and unoccupied static KS-orbitals and calculate approximations to the true excitation energies of the static many body system [3].

An alternative way is the explicit time propagation of "kicked orbitals" with the nonlinear TDDFT equations (23) and monitoring of certain observables. This is the key topic of this paper. 


\subsection{Kick perturbation}

Consider a $\delta(t)$ time dependent kick operator in the form of a spatial multiplication operator determined by $u: \mathbb{R}^{3} \rightarrow \mathbb{R}$

$$
\begin{aligned}
(\widehat{u} \psi)(\mathbf{x}) & =u(\mathbf{x}) \psi(\mathbf{x}) \\
\widehat{U}(t) & =\sum_{n=1}^{N} \widehat{u}^{(n)} \delta(t)
\end{aligned}
$$

Integral or differential operators would lead to instantaneous changes and a time discontinuity in the density and are therefore not considered here.

In the many body description the kick is described by

$$
\begin{gathered}
\Psi\left(t=0^{-}\right)=\Phi_{0} \stackrel{\widehat{U}(t)}{\rightarrow} \Psi\left(t=0^{+}\right)=\Psi_{0} \\
\Psi\left(\mathbf{x}_{1}, \ldots, \mathbf{x}_{N}, t=0^{+}\right)=\exp \left(-i \int_{0^{-}}^{0^{+}} \widehat{U}(t) d t\right) \Phi_{0}\left(\mathbf{x}_{1}, \ldots, \mathbf{x}_{N}\right)= \\
=e^{-i \sum_{n=1}^{N} u\left(\mathbf{x}_{n}\right)} \Phi_{0}\left(\mathbf{x}_{1}, \ldots, \mathbf{x}_{N}\right)=\left(\prod_{n=1}^{N} e^{-i u\left(\mathbf{x}_{n}\right)}\right) \Phi_{0}\left(\mathbf{x}_{1}, \ldots, \mathbf{x}_{N}\right)
\end{gathered}
$$

Note that the many-body density and therefore also the reduced densities are unchanged at the instant of the kick

$$
\rho^{(N)}\left(t=0^{+}\right)=\left|\Psi\left(t=0^{+}\right)\right|^{2}=\left|\Phi_{0}\right|^{2}=\rho^{(N)}\left(t=0^{-}\right) .
$$

\subsection{Type of kick function}

One has a freedom for the type of kick function $u$ which can be used to selectively excite the states one is particularly interested in. In particular we mention:

(1) cartesian: $u(\mathbf{x})=k_{n_{1} n_{2} n_{3}} x^{n_{1}} y^{n_{2}} z^{n_{3}}$

$$
u(\mathbf{x})=\mathbf{k} \cdot \mathbf{x}=k z
$$

(2) spherical: $u(\mathbf{x})=k_{n l m} r^{n} Y_{l m}(\omega)$

$$
u(\mathbf{x})=k|\mathbf{x}|=k r
$$

(3) cylindrical: $u(\mathbf{x})=k_{l m n} R^{l} y_{m}(\phi) z^{n}$

$$
u(\mathbf{x})=k R
$$

where $Y_{l m}, y_{m}$ are real $S^{2}, S^{1}$ spherical harmonics and a simple example of each class is given. Type (1) cartesian linear momentum kicks are the standard functions used for the long wavelength optical response. Corresponding to a spatially homogenous electric field $\mathbf{E}_{0}$ present only over a short time interval $[0, \Delta t]$ such that

$$
\begin{aligned}
& \qquad \begin{aligned}
\mathbf{E}(\mathbf{x}, \mathbf{t}) & =\mathbf{E}_{0}(t)=1_{[0, \Delta t]}(t) \mathbf{E}_{0} \\
\mathbf{E}_{0} \Delta t & =\mathbf{k}=\mathbf{c o n s t} . \\
\text { fieldstrength } * \text { duration } & =\text { momentum }=\text { const. }
\end{aligned}
\end{aligned}
$$

The electrostatic potential (in length gauge) is given by

$$
u(\mathbf{x}, t)=-\mathbf{E}_{0}(t) \cdot \mathbf{x}
$$


In the limit of $\Delta t \rightarrow 0, \mathbf{E}_{0} \rightarrow \infty, \mathbf{E}_{0} \Delta t=$ const. the external potential is given by

$$
\begin{aligned}
U(\mathbf{x}, t) & =-\lim _{\Delta \rightarrow 0}\left(\frac{1}{\Delta t}(\theta(t)-\theta(t-\Delta t))\right) \Delta t \mathbf{E} \cdot \sum_{n=1}^{N} \mathbf{x}_{n}= \\
& =-\delta(t) \mathbf{k} \cdot \sum_{n=1}^{N} \mathbf{x}_{n}=-\frac{1}{2 \pi} \int_{\mathbb{R}} 1 e^{i \omega t} d \omega \mathbf{k} \cdot \sum_{n=1}^{N} \mathbf{x}_{n}
\end{aligned}
$$

Physically that can be interpreted as a momentum transfer $\mathbf{k}$ at $t=0$ to each electron or an electric field with all frequencies according to the Fourier representation of the $\delta$ function [7].

\subsection{Further time evolution}

After the kick perturbation the time evolution of the many-body system is determined by the linear time-independent many particle Hamiltonian (1)

$$
\left\{\begin{array}{c}
i \frac{d}{d t} \Psi(t)=\widehat{H} \Psi(t) \\
\Psi(0)=\Psi_{0}
\end{array}\right.
$$

With the expansion into the eigenfunctions of the static problem (9) the time evolution is simply given by

$$
\begin{aligned}
& \Psi_{0}=\sum_{j} c_{j} \Phi_{j}=c_{0} \Phi_{0}+\sum_{j \geq 1} c_{j} \Phi_{j} \\
& \forall t>0: \Psi(t)=\sum_{j} c_{j} \Phi_{j}(t)=\sum_{j} c_{j} e^{-i E_{j} t} \Phi_{j}=c_{0} e^{-i E_{0} t} \Phi_{0}+\sum_{j \geq 1} c_{j} e^{-i E_{j} t} \Phi_{j} .
\end{aligned}
$$

If there is no relaxation mechanism included in the Hamiltonian, the system will (in general) never come back to the groundstate. The t-dependent (reduced one particle) density is given by

$$
\begin{aligned}
\rho(t) & =N \int d^{N-1} \mathbf{x}|\Psi(t)|^{2}=N \sum_{j, k} c_{j}^{*} c_{k} e^{i E_{j k} t} \int d^{N-1} \mathbf{x} \Phi_{j}^{*} \Phi_{k}= \\
& =N \sum_{j}\left|c_{j}\right|^{2} \int d^{N-1} \mathbf{x}\left|\Phi_{j}\right|^{2}+N \sum_{j \neq k} c_{j}^{*} c_{k} e^{i E_{j k} t} \int d^{N-1} \mathbf{x} \Phi_{j}^{*} \Phi_{k}
\end{aligned}
$$

Therefore the contributing frequencies are only the excitation energies of the system.

\subsection{Monitoring observables}

We also have the freedom which observables to monitor during the time evolution. Because of the TDDFT description we restrict ourselves to quantities which one can express with the reduced one body density. We take observables $a: \mathbb{R}^{3} \rightarrow \mathbb{R}$ 
and

$$
\begin{aligned}
\widehat{A}^{(N)} & =\sum_{n=1}^{N} a\left(\widehat{\mathbf{x}}_{n}\right) \\
A & :\left\{\begin{array}{l}
\mathbb{R}^{+} \rightarrow L^{1}\left(\mathbb{R}^{3}\right) \rightarrow \mathbb{R} \\
t \mapsto \rho_{t} \mapsto A(t)=\int a(\mathbf{x}) \rho_{t}(\mathbf{x}) d \mathbf{x}
\end{array}\right.
\end{aligned}
$$

in particular we consider the following classes with simple examples:

(1) cartesian multipole moments: $a(\mathbf{x})=x^{n_{1}} y^{n_{2}} z^{n_{3}}$

$$
a(\mathbf{x})=z
$$

(2) spherical multipole moments: $a(\mathbf{x})=r^{n} Y_{l m}(\omega)$

$$
a(\mathbf{x})=r
$$

(3) cylindrical multipole moments: $a(\mathbf{x})=R^{l} y_{m}(\phi) z^{n}$ $a(\mathbf{x})=R$

(4) local charges, characteristic functions: $\mathcal{V} \subseteq \mathbb{R}^{3}: a(\mathbf{x})=1_{\mathcal{V}}(\mathbf{x})$ $\mathcal{V}=B_{a}(\mathbf{0})=\{\mathbf{x}:|\mathbf{x}|<a\}$

For these types of monitoring observables we get the time dependence $A(t)$ by

$$
\begin{aligned}
A(t) & =\int_{\mathbb{R}^{3}} d \mathbf{x}(a(\mathbf{x}) \rho(\mathbf{x}, t))=N \sum_{j, k} c_{j}^{*} c_{k} e^{i\left(E_{j}-E_{k}\right) t} \int d^{N} \mathbf{x}\left(a\left(\mathbf{x}_{1}\right) \Phi_{j}^{*} \Phi_{k}\right)= \\
& =\sum_{j, k} c_{j}^{*} c_{k} e^{i E_{j k} t} \int d^{N} \mathbf{x}\left(a\left(\mathbf{x}_{1}\right)+\ldots+a\left(\mathbf{x}_{N}\right)\right) \Phi_{j}^{*} \Phi_{k}= \\
& =\sum_{j, k} c_{j}^{*} c_{k} e^{i E_{j k} t}<\Phi_{j}\left|\widehat{A}^{(N)}\right| \Phi_{k}>=\sum_{j, k} c_{j}^{*} c_{k} e^{i E_{j k} t}{ }_{a} I_{j k}= \\
& =\sum_{j}\left|c_{j}\right|^{2}{ }_{a} I_{j j}+\sum_{j \neq k} c_{j}^{*} c_{k} e^{i E_{j k} t}{ }_{a} I_{j k}
\end{aligned}
$$

where

$$
{ }_{a} I_{j k}=<\Phi_{j}\left|a\left(\widehat{\mathbf{x}}_{1}\right)+\ldots+a\left(\widehat{\mathbf{x}}_{N}\right)\right| \Phi_{k}>=<\Phi_{j}\left|\widehat{A}^{(N)}\right| \Phi_{k}>
$$

are static quantities, generalized oscillator strengths.

Therefore the t-dependence of $A(t)$ is determined by the excitation frequencies $E_{j k}$, the amplitudes with strengths $c_{k}$ are determined by the type of kick $u$ and the ${ }_{a} I_{j k}$ are determined by the monitoring observable $a$.

\subsection{Kick potential and further time evolution in TDDFT}

In the TDDFT approach we start with a static DFT calculation with the solution

$$
\left\{\begin{aligned}
\widehat{h}_{K S}[\rho] \varphi_{n} & =\varepsilon_{n} \varphi_{n}, n=1, \ldots, N \\
\rho(\mathbf{x}) & =\sum_{n=1}^{N}\left|\varphi_{n}(\mathbf{x})\right|^{2}
\end{aligned}\right.
$$

In TDDFT starting from the groundstate the xc-functional does not depend on the initial state.

Mathematically speaking TDDFT is a special, rather complicated case of a tdependent Nonlinear Schrödinger (NLS)-system [8], [9], [10] in the following called 
the TD-KS-NLS system.

$$
\left\{\begin{array}{l}
i \frac{d}{d t} \psi_{n}(t)=\widehat{h}_{K S}[\rho](t) \psi_{n}(t) \\
\psi_{n}(0)=\varphi_{n} \\
\rho(t)=\sum_{n=1}^{N}\left|\psi_{n}(t)\right|^{2} \\
\widehat{h}_{K S}[\rho](t)=\widehat{t}+\widehat{v}_{0}(t)+\widehat{v}_{H}[\rho](t)+\widehat{v}_{X C}[\rho](t) \\
\widehat{v}_{0}(t)=\widehat{v}_{0}+\widehat{u}(t) \text { external potential } \\
\widehat{v}_{H}[\rho](t), \widehat{v}_{X C}[\rho](t) \text { implicit t-dependence via } \rho\left(t^{\prime}\right), t^{\prime}<t
\end{array}\right.
$$

In our case the t-dependent part of the external potential is given by

$$
u(\mathbf{x}, t)=\delta(t) u(\mathbf{x})
$$

Therefore after the kick the orbitals are given by

$$
\psi_{n}\left(\mathbf{x}, 0^{+}\right)=e^{-i u(\mathbf{x})} \varphi_{n}(\mathbf{x})
$$

The charge density (as it should be) is not changed at the kick

$$
\rho\left(\mathbf{x}, 0^{+}\right)=\sum_{n=1}^{N}\left|\psi_{n}\left(\mathbf{x}, 0^{+}\right)\right|^{2}=\sum_{n=1}^{N}\left|\varphi_{n}(\mathbf{x})\right|^{2}=\rho_{0}(\mathbf{x})=\rho\left(\mathbf{x}, 0^{-}\right)
$$

Afterwards the kicked orbitals propagate with the (only implicitly) t-dependent KS-NLS equations

$$
\left\{\begin{array}{c}
i \frac{d}{d t} \psi_{n}(t)=\widehat{h}_{K S}[\rho](t) \psi_{n}(t) \\
\psi_{n}(\mathbf{x}, 0)=e^{-i u(\mathbf{x})} \varphi_{n}(\mathbf{x})
\end{array}\right.
$$

Here it is important to realize that the time dependence comes from the fact that $\psi_{n}(\mathbf{x}, 0)=e^{i \mathbf{k} \cdot \mathbf{x}} \varphi_{n}(\mathbf{x})$ is no longer an eigenfunction of $\widehat{h}_{K S}[\rho](t=0)=\widehat{h}_{K S}^{(0)}$.

Therefore $\rho$ will not remain constant in time, which implies that $\widehat{h}_{K S}$ becomes t-dependent and it is not the same as propagating with $\widehat{h}_{K S}^{(0)}$.

One can expand the kicked orbitals into the static KS-eigenfunctions (including unoccupied ones)

$$
\psi_{n}(\mathbf{x}, 0)=e^{i \mathbf{k} \cdot \mathbf{x}} \varphi_{n}(\mathbf{x})=\sum_{j} c_{j}^{(n)} \varphi_{j}(\mathbf{x})
$$

but because of the nonlinear evolution the time dependence is not given by pure phase factors

$$
\psi_{n}(\mathbf{x}, t) \neq \sum_{j} c_{j}^{(n)} e^{-i \varepsilon_{j} t} \varphi_{j}(\mathbf{x})
$$

The correct forward time propagation of the nonlinear KS equations leads to the t-dependent charge density

$$
\rho_{K S}(\mathbf{x}, t)=\sum_{n=1}^{N}\left|\psi_{n}(\mathbf{x}, t)\right|^{2}=\rho(\mathbf{x}, t)
$$


which is equal to the interacting true charge density (if an exact xc-functional would be known). Given an approximate t-dependent xc-functional we only get an approximation $\rho_{K S}(t)$. The KS-density $\rho_{K S}$ is used to calculate the t-dependent monitoring quantity $A(t)$

$$
A_{K S}(t)=\int d \mathbf{x}\left(a(\mathbf{x}) \rho_{K S}(\mathbf{x}, t)\right)
$$

The time-frequency analysis of $A(t)$ leads to the excitation energies and generalized transition strengths.

In the simplest case the Fourier transform of $A(t)$ has to be truncated to the finite time interval $[0, T]$ of the simulation and leads to

$$
\mathcal{F}[A](\omega) \approx \int_{0}^{T} A(t) e^{-i \omega t} d t
$$

out of which one can read off the excitation frequencies and the dipole strengths. An improvement is the smoothing of the signal at turn on (off) at $t=0(t=T)$ respectively. Alternatively the so called "harmonic inversion" [11] technique has been developed for these kind of signals, improving technical details of the signal analysis.

\section{Application to Helium excitation spectrum}

Unfortunately it is still widely unknown that one can straightforwardly reconstruct the exact KS potential for 2-body, spin singlet $S=0$ groundstates from data of correlated 2-body calculations (up to numerical precision). One can use among others MC-HF [12], Hylleraas-type variational or coupled spherical harmonics calculations. We used the results of [13] especially adapted to our radial grid.

The reconstruction schematically works in the following way:

$\Psi_{0}\left(\mathbf{x}_{1}, \mathbf{x}_{2}\right) \Rightarrow \rho(\mathbf{x}) \Rightarrow$ exact $v_{\mathrm{KS}}(\mathbf{x})$

The last step is done via

$$
v_{\mathrm{KS}}(\mathbf{x})=\frac{1}{2} \frac{\triangle\left(\rho(\mathbf{x})^{1 / 2}\right)}{\rho(\mathbf{x})^{1 / 2}}+\text { const. }
$$

For the spherical symmetric He density we get with

$$
\begin{gathered}
f(r)=r \varphi(r)=\left(r^{2} \frac{1}{2} \rho(r)\right)^{1 / 2} \\
v_{\mathrm{KS}}(r)=\frac{1}{2} \frac{f^{\prime \prime}(r)}{f(r)}+\text { const. }
\end{gathered}
$$

For didactical purposes one can subtract the nucleus and Hartree potential and plot the exact KS xc-potential for this particular system. Plotting $v_{x c}(r)$ and $\rho(r)$ shows the "nonlocality in space" of the exact xc potential. We refer for further aspects to the literature [14] and the recently published diploma work [15]. Be aware that for a numerical evaluation one does not have a closed form expression but only a finite number of radial points $\left(r_{j}, f\left(r_{j}\right)\right)_{j=1, \ldots, N}$. So one needs some interpolation and finite difference expressions for the derivatives. Careful numerics 


\begin{tabular}{|c|c|c|c|c|}
\hline KS-transition & $\begin{array}{c}\text { constructed } \\
\text { static } V_{K S}^{(K L I)}\end{array}$ & $\begin{array}{c}\text { reconstructed } \\
\text { static } V_{K S}^{(e x a c t)}\end{array}$ & $\begin{array}{c}\text { t-propagation } \\
V_{K S}^{(A K L I)}\end{array}$ & $\begin{array}{c}\text { Exact } \\
\text { nonrel. variational }\end{array}$ \\
\hline $1 s \rightarrow 2 s$ & 0.758 & 0.746 & 0.774 & 0.758 \\
$1 s \rightarrow 3 s$ & 0.852 & 0.839 & 0.856 & 0.843 \\
$1 s \rightarrow 4 s$ & 0.880 & 0.869 & 0.884 & 0.870 \\
$1 s \rightarrow 5 s$ & 0.896 & 0.883 & 0.896 & 0.883 \\
$1 s \rightarrow 6 s$ & 0.902 & 0.889 & 0.902 & 0.889 \\
\hline \hline $1 s \rightarrow 2 p$ & 0.788 & 0.777 & 0.778 & 0.780 \\
$1 s \rightarrow 3 p$ & 0.860 & 0.848 & 0.860 & 0.849 \\
$1 s \rightarrow 4 p$ & 0.884 & 0.872 & 0.888 & 0.873 \\
$1 s \rightarrow 5 p$ & 0.896 & 0.884 & 0.901 & 0.884 \\
$1 s \rightarrow 6 p$ & 0.902 & 0.890 & 0.907 & 0.890 \\
\hline
\end{tabular}

Table 1. KS static constructed and reconstructed, KS t-propagation and exact nonrelativistic variational transition energies. Columns with $V_{K S}^{(K L I)}$ and $V_{K S}^{(\text {exact })}$ are the bare KS eigenvalue differences of the static potential. Column $V_{K S}^{(A K L I)}$ are results from the kick/monitoring/FFT t-propagation method. The exact values of a nonrelativistic, variational calculation are from [20].

is needed because of the singularity at $r=0$ and the long range asymptotic

$$
\frac{f^{\prime \prime}(r)}{f(r)} \underset{r \rightarrow+\infty}{\longrightarrow} \frac{0}{0} \underset{r \rightarrow+\infty}{\longrightarrow}-\frac{1}{r}
$$

We use a cubic spline interpolation method and central difference formula for the derivative [16]. As usual we fix the constant by demanding $\lim _{r \rightarrow \infty} v_{\mathrm{KS}}=0$ and use $\forall r>15$ a.u. $v_{\mathrm{KS}}=-\frac{1}{r}$.

Out of the exact static $v_{K S}$ we calculate the exact KS-eigenvalues and KS excitation energies. This was done in a standard way of numerically solving the eigenvalue problem with a shooting method and Numerov $O\left(h^{6}\right)$ method for the ODE-IVP. Collocation and other techniques might be used as well [17].

The results are presented together with the other data in table 1.

We compare the KS-eigenvalue differences of the exactly reconstructed static $v_{K S}$ and the constructed $v_{K S}^{O E P-E X-K L I}$ potential. The constructed KS-potential used was the KLI approximation of the OEP-EX [18] within Qprop [1].

For the t-dependent calculation one needs an approximation for the $t$-dependent xc-potential as explained in this paper, for which we use adiabatic OEP-EX-KLI, AKLI $v_{x c}^{(A K L I)}$.

In this work we focus on the explicit t-propagation method, results from linear response-TDDFT can be found in [19].

The results of the $1 s \rightarrow n p \mathrm{KS}$ excitations, corresponding to $1^{1} S \rightarrow n^{1} P$ excitations of the interacting system are pretty standard and can be well calculated with standard linear momentum kicks and dipole moment monitoring. The $1^{1} S \rightarrow n^{1} S$ excitations are calculated by different kick/monitoring methods and the Fourier analyzed signals are shown in fig. 1, the corresponding energies can be found in table 1. We use a radial kick, see section (3.3) type (2) and monitor both the radial quadrupole moment and the local charge within a ball of radius $a={ }_{2 s} r_{1}$ equal to the first node of the 2 s static KS orbital. For this "radial breathing mode", excited by this specific kick, both monitoring quantities are well adapted and lead to sharp frequency peaks in the FFT [21] signal.

As already known in the literature the excitation energies are rather sensitive to the static KS orbitals and therefore t-propagation methods can not compete with linear response-TDDFT methods when a reconstructed exact KS potential is used. A method for reconstruction of the t-dependent KS potential for 2 parti- 


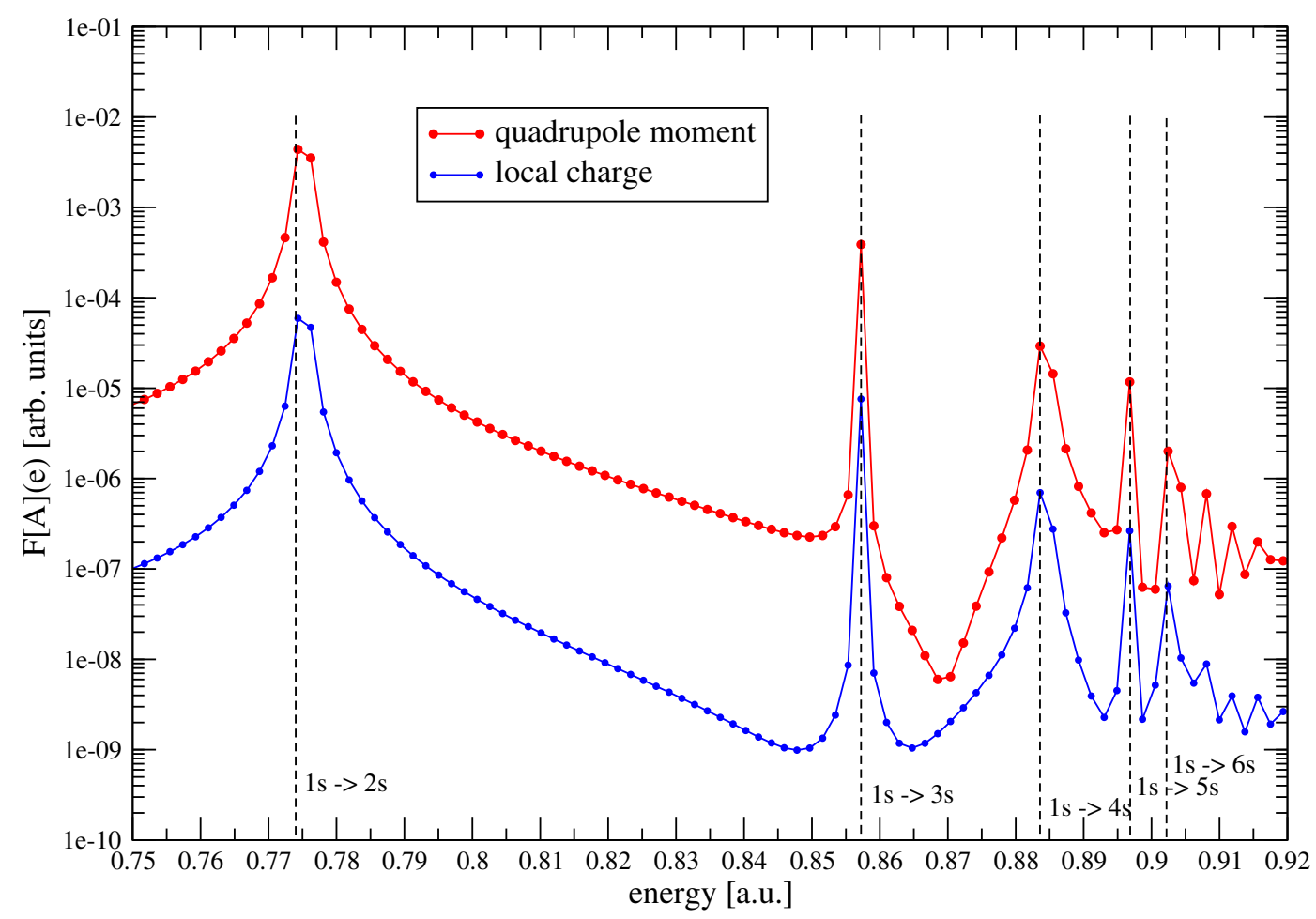

Figure 1. $\quad 1^{1} S \rightarrow \mathrm{n}{ }^{1} S$ transition energies calculated from Fourier analyses of quadrupole moment and local charge as monitoring signals. Be aware of the semi-logarithmic scale.

cles in $3 d$ is not known. For Helium even bare static KS eigenvalue differences are already excellent approximations to true excitation energies. Adiabatic OEP-EXKLI systematically overestimates excitation energies and shifts transition energies to higher frequencies. The good agreement between static KS-KLI excitation energies and exact values is rather due to a cancellation of errors. Reconstructed static KS excitations are below the true singlet values, t-propagation methods shift them upwards.

\section{Conclusion}

Explicit time propagation methods for the calculation of excitation energies are extended to generalized kick perturbations and monitoring observables. While linear response-TDDFT methods are favorable compared to explicit t-propagation for small systems, explicit t-propagation has its advantages if unoccupied KS states are expensive to calculate and for bigger systems. Physical insight coming from t-dependent local charges within certain regions of the system will mainly be valuable for molecules. A selective excitation of a certain region of the molecule and the following probing of the t-dependent response is possible. Also without kick perturbations but during a real time evolution of the molecular charge density in a laser field one can study the 'charge stiffness' from monitoring t-dependent local charges. Work on molecules along these lines is in progress.

\section{Acknowledgments}

This work was supported by the Austrian Ministry of Science via its grant for the Wolfgang Pauli Institute and by the City of Vienna Science and Technology Fund 
(WWTF) via the project "Mathematik und 2004" MA-04-45 and "Mathematik und 2007" MA-07-11.

\section{References}

[1] Dieter Bauer and Peter Koval. Qprop: A schrodinger-solver for intense laser-atom interaction. Computer Physics Communications, 174, 5:396, 2006.

[2] Erich Runge and E. K. U. Gross. Density-functional theory for time-dependent systems. Phys. Rev. Lett., 52(12):997, Mar 1984.

[3] M.A.L. Marques and E.K.U. Gross. Time-dependent density functional theory. Annual Review of Physical Chemistry, 55(1):427-455, 2004.

[4] Andreas Görling. Density-functional theory for excited states. Phys. Rev. A, 54(5):3912-3915, Nov 1996.

[5] H. Appel, E. K. U. Gross, and K. Burke. Excitations in time-dependent density-functional theory. Phys. Rev. Lett., 90(4):043005, Jan 2003.

[6] M.A. L.Marques, C.A. Ullrich, F. Nogueira, A. Rubio, K. Burke, and E.K.U. Gross. Time-Dependent Density Functional Theory. Springer-Verlag.

[7] P. M. Morse and H. Feshbach. Methods of theoretical physics. International Series in Pure and Applied Physics, New York: McGraw-Hill, 1953, 1953.

[8] J. Ginibre. An introduction to nonlinear Schrödinger equations. GAKUTO Int. Ser., Math. Sci. Appl. 10, 85-133(1997)., 1997.

[9] H. P. Stimming and N.Mauser. Lecture notes: Nonlinear schrödinger equations. Uni Wien lecture notes.

[10] Weizhu Bao, N.J. Mauser, and H.P. Stimming. Effective one particle quantum dynamics of electrons: a numerical study of the Schrödinger-Poisson-X $\alpha$ model. Commun. Math. Sci., 1(4):809-828, 2003.

[11] Vladimir A. Mandelshtam and Howard S. Taylor. Harmonic inversion of time signals and its applications. The Journal of Chemical Physics, 107(17):6756-6769, 1997.

[12] Armin Scrinzi and Bernard Piraux. Two-electron atoms in short intense laser pulses. Phys. Rev. A, 58(2):1310-1321, Aug 1998.

[13] J. Feist, S. Nagele, R. Pazourek, E. Persson, B. I. Schneider, L. A. Collins, and J. Burgdorfer. Nonsequential two-photon double ionization of helium. Physical Review A (Atomic, Molecular, and Optical Physics), 77(4):043420, 2008.

[14] R. van Leeuwen and E. J. Baerends. Exchange-correlation potential with correct asymptotic behavior. Phys. Rev. A, 49(4):2421-2431, Apr 1994.

[15] L. Schimka. Interaction of atoms with laser fields using TDDFT within the Qprop software. PhD thesis, TU-Wien diploma thesis, 2008.

[16] H. R. Schwarz. Numerische Mathematik. B. G. Teubner, Stuttgart, 1997.

[17] W. Auzinger, O. Koch, and E. Weinmüller. Efficient collocation schemes for singular boundary value problems. Numerical Algorithms, 31(1):5-25, 2002.

[18] T. Grabo and E. K. U. Gross. The optimized effective potential method of density functional theory: Applications to atomic and molecular systems. International Journal of Quantum Chemistry, 64, 1:95, 1997.

[19] M. Petersilka, E.K.U.Gross, and Kieron Burke. Excitation energies from time-dependent density functional theory using exact and approximate potentials. International Journal of Quantum Chemistry, 80(4-5):534-554, 2000.

[20] Akihiro Kono and Shuzo Hattori. Accurate oscillator strengths for neutral helium. Phys. Rev. A, 29(6):2981-2988, Jun 1984.

[21] M. Frigo, S.G.Johnson., and other FFTW developers. Fftw software. http://www.fftw.org/, 2005. 


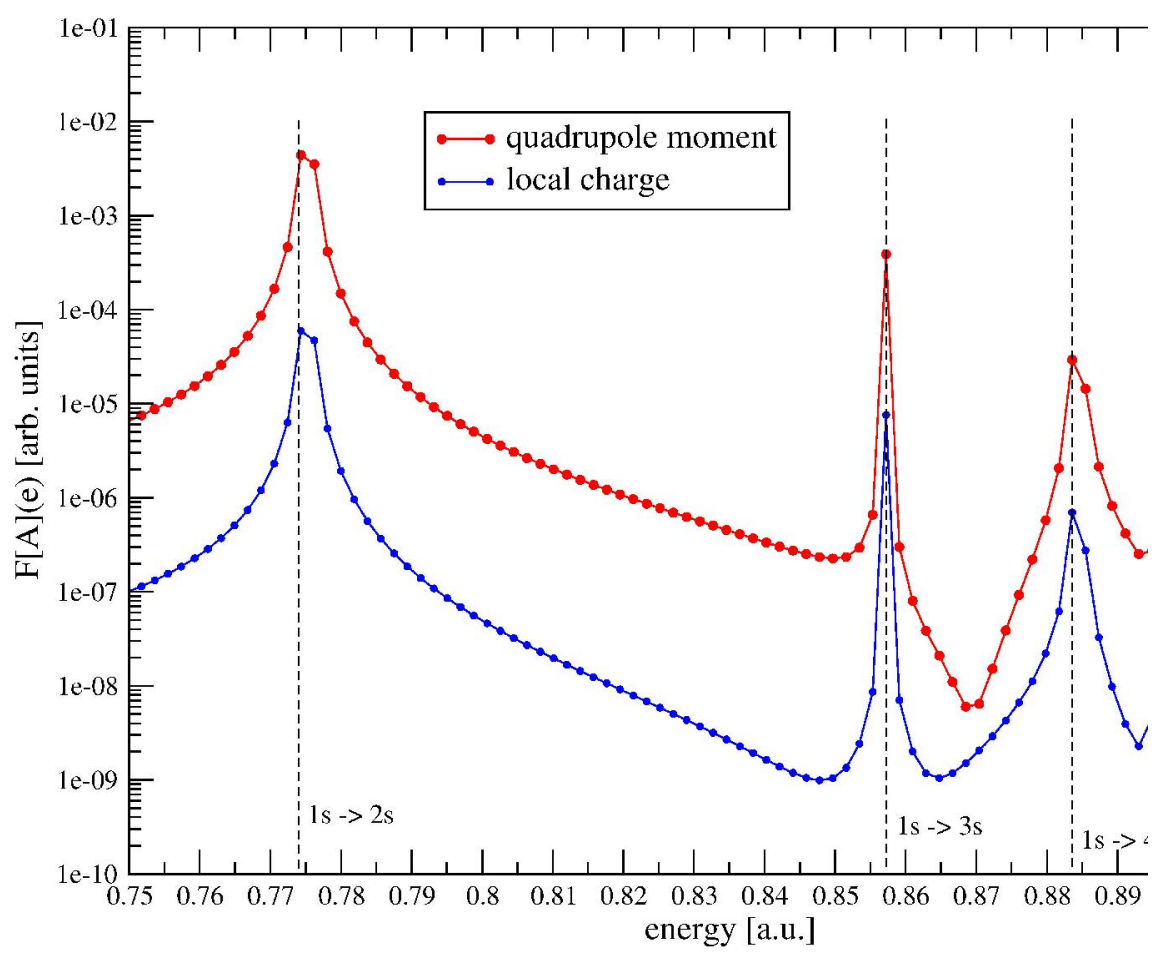

Figure 1

$215 \times 279 \mathrm{~mm}(600 \times 600 \mathrm{DPI})$ 


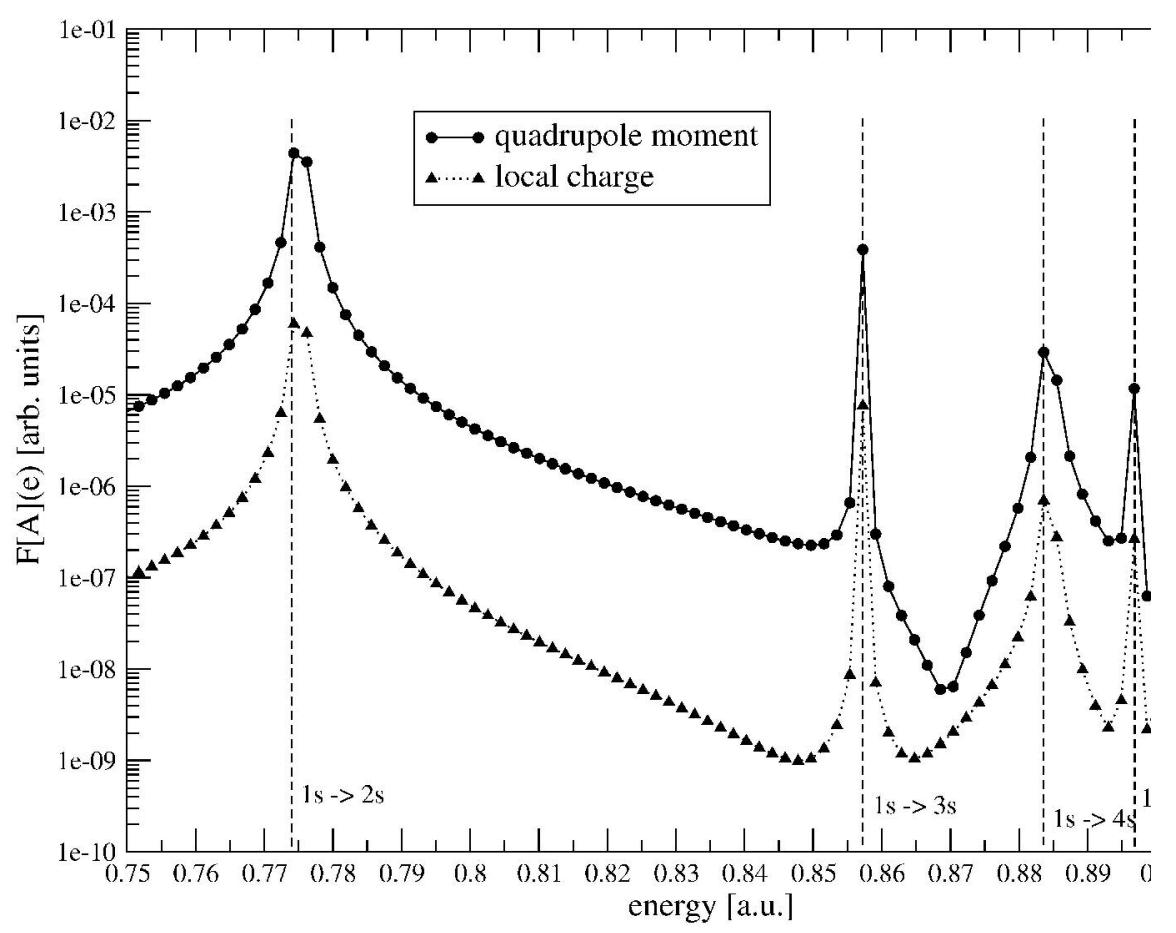

Figure 1

$215 \times 279 \mathrm{~mm}(600 \times 600 \mathrm{DPI})$ 